\title{
Segurança do paciente idoso hospitalizado: uma análise do risco de quedas
}

\author{
Safety of hospitalized older adult patients: an analysis of the risk of falls \\ Seguridad de los pacientes ancianos hospitalizados: un análisis del riesgo de caídas
}

Como citar este artigo:

Canuto CPAS, Oliveira LPBA, Medeiros MRS, Barros WCTS. Safety of hospitalized older adult patients: an analysis of the risk of falls. Rev Esc Enferm USP. 2020;54:e03613. doi: https://doi.org/10.1590/S1980-220X2018054003613

\section{Carla Patrícia de Almeida Santos Canuto $^{1}$ \\ (D) Luciane Paula Batista Araujo de Oliveira $^{1}$ \\ Marília Rute de Souto Medeiros ${ }^{1}$ \\ Wanessa Cristina Tomaz dos Santos Barros ${ }^{1}$ \\ ${ }^{1}$ Universidade Federal do Rio Grande do Norte, Faculdade de Ciências da Saúde do Trairi, Santa Cruz, RN, Brasil.}

\begin{abstract}
Objective: To identify the risk of falls of older adults in a hospital in the Trairi region in Rio Grande do Norte, Brazil, and to describe the relationship between risk of falls and the sociodemographic characteristics of the participants. Method: A descriptive, transversal, and quantitative study conducted in a regional hospital, respecting the ethical principles in force. The Morse Scale was adopted for data collection, which took place in July and August 2018, and adapted with sociodemographic questions. A descriptive and inferential statistical analysis was performed. Results: There were 46 participants in the study, most of whom were women with low education, and the most frequent reasons for hospitalization were surgical treatment and lung disease. More than half had a high risk of falling $(54.35 \%)$, followed by moderate $(32.61 \%)$ and low $(13.04 \%)$. There was an association between high risk of falls, having lung disease as a reason for hospitalization and diabetes as a comorbidity. The high risk of falls was less frequent among older adult patients hospitalized for surgical treatment. Conclusion: The high risk of falls was found in more than half of the older adults, which varied according to the reason for hospitalization and comorbidities, being more frequent in those hospitalized for lung disease and in those who had Diabetes.
\end{abstract}

DESCRIPTORS

Aged; Hospitalization; Accidental Falls; Patient Safety; Geriatric Nursing. 


\section{INTRODUÇÃO}

Segundo a Organização Mundial da Saúde (OMS), o conceito de Segurança do Paciente se refere à redução dos riscos de danos desnecessários associados à assistência em saúde até um mínimo aceitável ${ }^{(1)}$. Esse assunto é importante na prevenção de agravos aos pacientes por estimular a realização de medidas básicas como, por exemplo, a identificação correta do paciente, a higiene das mãos, o uso de protocolos institucionais, entre outras.

A Aliança Mundial para a Segurança do Paciente, lançada em 2004 pela OMS, tem como objetivo facilitar o desenvolvimento de políticas e práticas para a segurança do paciente. O Brasil deu um passo importante nesse sentido ao instituir o Programa Nacional de Segurança do Paciente por meio do Ministério da Saúde ${ }^{(2)}$.

A Rede Brasileira de Enfermagem e Segurança do Paciente (REBRAENSP) elaborou em 2013 um manual com 12 estratégias baseadas nos desafios globais formulados pela Aliança Mundial de Segurança do paciente, visando à prevenção de danos e promoção da saúde. Dentre essas estratégias, destaca-se neste estudo aquela que aborda a prevenção de quedas ${ }^{(2)}$.

No Brasil, a Portaria no 2.095, de 24 de setembro de 2013, aprovou os protocolos básicos de segurança do paciente, dentre estes, o Protocolo de Prevenção de Quedas, que determina a necessidade de avaliar todos os pacientes quanto ao risco desse evento, seguido pela elaboração e prescrição de medidas preventivas de acordo com o risco de cada indivíduo ${ }^{(3)}$.

Queda define-se como deslocamento involuntário do corpo para um nível inferior à posição inicial, provocado por múltiplos fatores, resultando ou não em dano ao paciente. A queda pode ocorrer da própria altura, da maca/cama ou de assentos como cadeira de rodas, poltronas, cadeiras, banheira, cadeira higiênica ou vaso sanitário ${ }^{(3-4)}$.

Para os idosos em ambiente hospitalar, a ocorrência de quedas pode estar associada a fatores como uso contínuo de medicamentos, déficits sensoriais - visual, auditivo, tátil -, uso de óculos e de dispositivos de auxílio para locomoção, como bengala, cadeira de rodas ou andador ${ }^{(5)}$.

$\mathrm{O}$ evento quedas pode ter repercussões mais sérias para a saúde da pessoa idosa, pois o processo natural de envelhecimento humano acarreta redução da densidade óssea, da massa muscular e da força física. Estes parâmetros refletem na postura, na deambulação, no equilíbrio e em fatores que aumentam o risco do evento quedas ${ }^{(6)}$.

Acidentes por queda são a principal causa de lesões não fatais e de morte em idosos e, por isso, se constituem um grave problema de saúde pública ${ }^{(7)}$ cujos impactos merecem maior atenção não somente no âmbito das pesquisas, como também na assistência à saúde e políticas públicas. Outros autores encontraram que alguns profissionais da saúde reduzem a atenção à ocorrência das quedas por considerá-las apenas como desdobramentos inevitáveis do envelhecimento ${ }^{(8)}$.

Nesse sentido, a vigilância para a prevenção da ocorrência de quedas deve ser também uma das prioridades da assistência de enfermagem no momento da internação do paciente na instituição de saúde. A identificação do risco de quedas fornece informações fundamentais para a formulação de planos de cuidados mais adequados às necessidades individuais de cada paciente, dando subsídios para a implementação da Sistematização da Assistência de Enfermagem ${ }^{(9)}$.

No período de 1996 a 2012, ocorreram 941.923 internações com diagnóstico secundário associado a queda entre pessoas idosas no Brasil. Além disso, as taxas de internação nessa faixa etária continuam elevadas, por isso, devem ser lembradas não só as repercussões físicas como também as consequências biopsicossociais causadas pelas quedas ${ }^{(10)}$, que incluem sofrimento, hospitalização, isolamento - suscitando questionamentos que poderão conduzir à elaboração de propostas para prevenção deste evento nessa população. Além disso, as quedas são um dos indicadores mais importantes para a segurança do paciente, por isso, o Ministério da Saúde e o Programa Nacional de Segurança do Paciente incluem a avaliação do risco de quedas como uma das intervenções mais efetivas na prevenção desse evento adverso.

Sendo assim, este estudo buscou responder a seguinte questão de pesquisa: Qual o risco de acidentes por queda em pacientes idosos internados em um hospital da região Trairi do Estado do Rio Grande do Norte?

Diante do exposto, teve como objetivos: identificar o risco de quedas em idosos em um hospital da região do Trairi, no Rio Grande do Norte; descrever a relação entre risco de quedas e as características sociodemográficas dos participantes.

\section{MÉTODO}

\section{TIPO DE ESTUDO}

Estudo do tipo descritivo, com recorte transversal e de abordagem quantitativa.

\section{Cenário}

O estudo foi realizado em um hospital da região Trairi, localizado no Município de Santa Cruz, Região do Trairi, no Estado do Rio Grande do Norte.

Esse hospital possui 40 leitos, sendo considerado de pequeno porte, e atende pacientes oriundos dos 11 municípios da região do Trairi. A escolha do local ocorreu devido à importância do mesmo no atendimento clínico geral e de urgência na região, para todos os públicos, principalmente para os pacientes idosos, além de situar-se em uma cidade que tem a presença de um campus da Universidade Federal do Rio Grande do Norte, com quatro cursos de graduação na área da saúde - enfermagem, fisioterapia, nutrição e psicologia.

Para o curso de enfermagem, o hospital serve como campo para as atividades práticas da disciplina de semiologia e semiotécnica e estágio supervisionado, oportunizando o desenvolvimento de habilidades e competências importantes para a formação do profissional enfermeiro. Atualmente, o hospital não possui núcleo de segurança do 
paciente, um serviço de extrema importância para promover a prevenção, controle e mitigação de incidentes, além da integração dos setores, promovendo a articulação dos processos de trabalho e das informações que impactam nos riscos ao paciente.

\section{CRItérios de Seleção}

Os critérios de inclusão foram: ter idade igual ou superior a 60 anos; estar internado nos setores de clínica médica ou cirúrgica do hospital supracitado no período de coleta; e ser capaz de compreender as perguntas dos instrumentos. Foram excluídos aqueles que apresentavam dificuldades de comunicação que os impedissem de responder aos instrumentos ou aqueles cujo estado de saúde fosse grave e/ou instável, evitando assim desconfortos durante a coleta.

Para delimitação da amostra, partiu-se do número de internações de idosos ocorridas nos meses de julho e agosto de 2017 , tendo em vista que correspondia ao mesmo período em que viria a ser realizada a coleta de dados no ano de 2018. Assim, para o cálculo amostral foram considerados uma população de 52 idosos, intervalo de confiança de $95 \%$ e a frequência estimada de $52 \%$, que foi o percentual de idosos com risco de quedas elevado em estudo realizado com idosos em Belém/Pará ${ }^{(11)}$. Dessa forma, a amostra calculada para a realização deste estudo foi de 46 idosos.

\section{Coleta de dados}

A técnica de coleta de dados foi a entrevista do tipo estruturada, tendo como instrumento a Escala de Morse, a qual contém questões fechadas que abordam histórico de quedas, diagnósticos secundários, auxílio na deambulação, uso de terapia endovenosa/dispositivo endovenoso salinizado ou heparinizado, marcha e estado mental. Foi utilizada a versão do instrumento traduzido e validado por outros autores $^{(12)}$ em que a pontuação da escala pode variar de $0 \mathrm{a}$ 125 pontos, sendo de 0 a 24 pontos considerado Risco Baixo, de 25 a 44 pontos como Risco Moderado, e $\geq 45$ pontos considera-se Risco Alto.

No cabeçalho desse instrumento foram inseridas algumas questões referentes aos dados sociodemográficos dos participantes, como sexo, faixa etária, escolaridade (em anos), motivo de internação, tempo de internação (em dias) e doenças autorrelatadas. Essas questões sociodemográficas foram obtidas da consulta ao prontuário individual dos idosos. Do prontuário, também foram retiradas as informações para preencher os itens 1 e 2 da Escala de Morse; os demais itens da escala foram avaliados junto ao participante na enfermaria em que se encontrava internado e registrados no formulário da pesquisa.

\section{ANÁLISE E TRATAMENTO DOS DADOS}

O banco de dados foi construído em planilha do programa EXCEL, versão 2017; para realização das tabelas descritivas e aplicação de testes estatísticos utilizou-se o software Statistical Package for the Social Sciences (SPSS ${ }^{\circledR}$ ), versão 25.0.

Para as variáveis quantitativas avaliadas no estudo, analisaram-se estatísticas descritivas de medidas de tendência e de dispersão dos dados, como, por exemplo: mínimo, máximo, média e desvio padrão. Para variáveis qualitativas, realizou-se análise descritiva por meio de distribuições de frequências absolutas e relativas (\%), tendo como valor de $\mathrm{p} \leq 0,05$.

$\mathrm{Na}$ comparação da classificação do risco de queda de Morse, quanto ao perfil geral do paciente com as variáveis categorizadas, aplicou-se o teste estatístico Qui-Quadrado. $\mathrm{Na}$ realização de todos os testes estatísticos foi considerado o nível de significância de 5\%, adotando o p-valor $\leq 0,05$.

\section{Aspectos éticos}

A pesquisa respeitou todos os princípios éticos da Resolução no 466/2012, do Conselho Nacional de Saúde. O protocolo de pesquisa foi apreciado e aprovado pelo Comitê de Ética em Pesquisa da Faculdade de Ciências da Saúde do Trairi/Universidade Federal do Rio Grande do Norte, por meio do Parecer $n^{\circ}$ 2.715.284/2018. Todos tiveram acesso ao Termo de Consentimento Livre Esclarecido (TCLE) e aqueles que concordaram em participar da pesquisa assinaram o termo antes de iniciar os procedimentos de coleta.

\section{RESULTADOS}

A média de idade dos participantes foi de 75,02 anos, com desvio padrão de 10,08 anos, variando de 60 a 104 anos de idade. Metade dos participantes era não alfabetizada e a maioria dos entrevistados era do sexo feminino. Os principais motivos de internação desses idosos foram tratamento cirúrgico e doença pulmonar. Informações adicionais sobre a caracterização dos participantes se encontram na Tabela 1.

Tabela 1 - Caracterização dos participantes quanto ao sexo, faixa etária, escolaridade (em anos), motivos de internação e comorbidades - Santa Cruz, RN, Brasil, 2018.

\begin{tabular}{lcrr}
\hline Variáveis & Características & N & \% \\
\hline \multirow{2}{*}{ Sexo } & Feminino & 26 & 56,52 \\
& Masculino & 20 & 43,48 \\
\hline \multirow{2}{*}{ Faixa etária } & & $\mathbf{4 6}$ & $\mathbf{1 0 0 , 0 0}$ \\
& Até 75 anos & 27 & 58,70 \\
\hline
\end{tabular}


...continuação

\begin{tabular}{|c|c|c|c|}
\hline Variáveis & Características & $\mathbf{N}$ & $\%$ \\
\hline \multirow{7}{*}{$\begin{array}{l}\text { Escolaridade } \\
\text { (em anos) }\end{array}$} & Não alfabetizado & 23 & 50,00 \\
\hline & $1-3$ anos & 15 & 32,61 \\
\hline & $4-7$ anos & 5 & 10,88 \\
\hline & $8-11$ anos & 1 & 2,17 \\
\hline & Acima de 11 anos & 1 & 2,17 \\
\hline & Sem resposta & 1 & 2,17 \\
\hline & & 46 & 100,00 \\
\hline \multirow{10}{*}{ Motivos de internação } & Tratamento cirúrgico & 15 & 32,61 \\
\hline & Doença pulmonar & 12 & 26,09 \\
\hline & Doença infecciosa & 7 & 15,22 \\
\hline & Doença renal & 4 & 8,70 \\
\hline & Doença cardíaca & 4 & 8,70 \\
\hline & Septicemia & 1 & 2,17 \\
\hline & Doença circulatória & 1 & 2,17 \\
\hline & Pancreatite & 1 & 2,17 \\
\hline & Gastroenterite aguda & 1 & 2,17 \\
\hline & & 46 & 100,00 \\
\hline
\end{tabular}

Nota: $(\mathrm{n}=46)$.

Os participantes apresentaram uma média de 1,93 dias de internação e tiveram até três comorbidades por pessoa (Tabela 2). Sobre as comorbidades, 29 participantes referiram ter Hipertensão Arterial Sistêmica (HAS), 13 tinham Diabetes (DM), três casos de Acidente Vascular Encefálico, um de Doença Pulmonar Obstrutiva Crônica e seis mencionaram outras doenças; nove participantes não apresentaram nenhuma comorbidade.

A Tabela 3 apresenta as frequências dos itens da Escala de Morse que compõem a avaliação do risco de quedas.

Tabela 2 - Descrição das variáveis dos pacientes quanto à idade (em anos), tempo de internação e número de comorbidades - Santa Cruz, RN, Brasil, 2018.

\begin{tabular}{lccccccccc}
\hline Variáveis & Mín. & Máx. & $\mathbf{2 5} \%$ & Mediana & $\mathbf{7 5} \%$ & Média & DP & CV & p-valor* \\
\hline $\begin{array}{l}\text { Idade } \\
\text { (em anos) }\end{array}$ & 60,00 & 104,00 & 66,00 & 74,00 & 82,00 & 75,02 & 10,08 & 13,43 & 0,033 \\
$\begin{array}{l}\text { Tempo de internação } \\
\text { (em dias) }\end{array}$ & 1,00 & 9,00 & 1,00 & 1,00 & 2,00 & 1,93 & 1,68 & 86,76 & $<0,001$ \\
№ de doenças & 0,00 & 3,00 & 1,00 & 1,00 & 1,00 & 1,07 & 0,71 & 66,83 & $<0,001$ \\
\hline
\end{tabular}

Nota: $(n=46)$.

Legenda: DP: Desvio Padrão CV: Coeficiente de Variação

${ }^{*}$ p-valor $\leq 0,05$ (aplicado teste de Shapiro-Wilk para verificar a normalidade dos dados)

Tabela 3 - Frequência de respostas aos itens da Escala de Morse - Santa Cruz, RN, Brasil, 2018.

\begin{tabular}{lccc}
\hline Itens & Respostas & Frequência & \% \\
\hline \multirow{2}{*}{ Históricos de quedas } & Não & 35 & 76,09 \\
& Sim & 11 & 23,91 \\
Diagnóstico secundário & Não & 19 & 41,30 \\
& Sim & 27 & 58,70 \\
Auxílio na deambulação & Nenhum/Acamado/Auxiliado por Profissional da Saúde & 45 & 97,83 \\
& Muletas/Bengala/Andador & 1 & 2,17 \\
\hline
\end{tabular}


...continuação

\begin{tabular}{|c|c|c|c|}
\hline Itens & Respostas & Frequência & $\%$ \\
\hline \multirow{2}{*}{ Uso de dispositivo endovenoso } & Não & 1 & 2,17 \\
\hline & Sim & 45 & 97,83 \\
\hline \multirow{3}{*}{ Marcha } & Normal/Sem deambulação, Acamado, Cadeira de Rodas & 23 & 50,00 \\
\hline & Fraca & 20 & 43,48 \\
\hline & Comprometida/Cambaleante & 3 & 6,52 \\
\hline \multirow{2}{*}{ Estado mental } & Orientado/capaz quanto à sua capacidade/limitação & 34 & 73,91 \\
\hline & Superestima capacidade/Esquece limitações & 12 & 26,09 \\
\hline
\end{tabular}

Nota: $(\mathrm{n}=46)$.

$\mathrm{Na}$ avaliação da classificação geral da escala de risco de quedas houve predominância de risco alto, conforme apresentado na Tabela 4.

Quanto às associações entre variáveis, foi identificado que os pacientes que tiveram doença pulmonar como motivo de internação e os que apresentaram a comorbidade Diabetes Mellitus apresentaram maior percentual de risco de queda alto. Isso reforça a importância do acompanhamento e cuidado contínuo dos idosos, visto que tais doenças e suas complicações são consideradas condições sensíveis à Atenção Primária à Saúde, ou seja, se tratadas nesse nível de atenção, poderiam evitar internações hospitalares futuras e, com isso, o risco de quedas vivido nesse cenário de assistência à saúde. Apesar de não haver associação estatística, destaca-se que mais de $60 \%$ dos idosos eram hipertensos e destes, quase $70 \%$ tinham risco moderado ou elevado para queda. Além disso, os pacientes cujo motivo de internação foi tratamento cirúrgico apresentaram menor percentual de risco de queda alto (Tabela 5).

Tabela 4 - Classificação quanto ao risco de quedas - Santa Cruz, RN, Brasil, 2018.

\begin{tabular}{lll}
\hline Classificação de risco de quedas segundo a Escala de Morse & $\mathbf{n}$ & $\%$ \\
\hline Risco baixo & 6 & 13,04 \\
Risco moderado & 15 & 32,61 \\
Risco alto & 25 & 54,35 \\
\hline
\end{tabular}

Nota: $(n=46)$.

Tabela 5 - Associação entre o risco de queda e a caracterização dos participantes - Santa Cruz, RN, Brasil, 2018.

\begin{tabular}{|c|c|c|c|c|c|c|}
\hline \multirow{2}{*}{\multicolumn{2}{|c|}{ Características gerais }} & \multicolumn{3}{|c|}{ Risco } & \multirow{2}{*}{$\begin{array}{c}\text { Total } \\
\%\end{array}$} & \multirow{2}{*}{ p-valor* } \\
\hline & & Baixo & Moderado & Alto & & \\
\hline \multirow{2}{*}{ Sexo } & Feminino & $\begin{array}{l}7,69 \\
(n=2)\end{array}$ & $30,77(\mathrm{n}=8)$ & $\begin{array}{l}61,54 \\
(n=16)\end{array}$ & $\begin{array}{l}100,00 \\
(\mathrm{n}=26)\end{array}$ & \multirow{2}{*}{0,176} \\
\hline & Masculino & $\begin{array}{l}20,00 \\
(n=4)\end{array}$ & $35,00(n=7)$ & $\begin{array}{l}45,00 \\
(n=9)\end{array}$ & $\begin{array}{l}100,00 \\
(n=20)\end{array}$ & \\
\hline \multirow{2}{*}{ Idade } & Até 75 anos & $\begin{array}{l}14,82 \\
(n=4)\end{array}$ & $40,74(n=11)$ & $\begin{array}{l}44,44 \\
(\mathrm{n}=12)\end{array}$ & $\begin{array}{l}100,00 \\
(n=27)\end{array}$ & \multirow{2}{*}{0,188} \\
\hline & Acima de 75 anos & $\begin{array}{l}10,53 \\
(n=2)\end{array}$ & $21,05(n=4)$ & $\begin{array}{l}68,42 \\
(n=13)\end{array}$ & $\begin{array}{l}100,00 \\
(n=19)\end{array}$ & \\
\hline \multirow{2}{*}{$\begin{array}{l}\text { Tempo de } \\
\text { internação }\end{array}$} & Até 2 dias & $\begin{array}{l}15,38 \\
(n=6)\end{array}$ & $30,77(n=12)$ & $\begin{array}{l}53,85 \\
(\mathrm{n}=21)\end{array}$ & $\begin{array}{l}100,00 \\
(\mathrm{n}=39)\end{array}$ & \multirow{2}{*}{0,526} \\
\hline & Acima de 2 dias & $\begin{array}{l}0,00 \\
(n=0)\end{array}$ & $42,86(n=3)$ & $\begin{array}{l}57,14 \\
(\mathrm{n}=4)\end{array}$ & $\begin{array}{c}100,00 \\
(n=7)\end{array}$ & \\
\hline \multirow{2}{*}{$\begin{array}{l}\text { Tempo de } \\
\text { escolaridade }\end{array}$} & Sim & $\begin{array}{l}15,79 \\
(n=6)\end{array}$ & $34,21(n=13)$ & $\begin{array}{l}50,00 \\
(\mathrm{n}=19)\end{array}$ & $\begin{array}{l}100,00 \\
(\mathrm{n}=38)\end{array}$ & \multirow{2}{*}{0,209} \\
\hline & Não & $\begin{array}{c}0,0 \\
(n=0)\end{array}$ & $28,57(\mathrm{n}=2)$ & $\begin{array}{l}71,43 \\
(\mathrm{n}=5)\end{array}$ & $\begin{array}{c}100,00 \\
(n=7)\end{array}$ & \\
\hline \multirow{2}{*}{$\begin{array}{l}\text { Tratamento } \\
\text { Cirúrgico }\end{array}$} & Sim & $\begin{array}{r}33,33 \\
(n=5)\end{array}$ & $53,34(\mathrm{n}=8)$ & $\begin{array}{l}13,33 \\
(\mathrm{n}=2)\end{array}$ & $\begin{array}{l}100,00 \\
(n=15)\end{array}$ & \multirow{2}{*}{$<0,001$} \\
\hline & Não & $\begin{array}{l}3,23 \\
(n=1)\end{array}$ & $22,58(n=7)$ & $\begin{array}{l}74,19 \\
(\mathrm{n}=23)\end{array}$ & $\begin{array}{l}100,00 \\
(n=31)\end{array}$ & \\
\hline
\end{tabular}


...continuação

\begin{tabular}{|c|c|c|c|c|c|c|}
\hline \multirow{2}{*}{ Características gerais } & & \multicolumn{3}{|c|}{ Risco } & \multirow{2}{*}{$\begin{array}{c}\text { Total } \\
\%\end{array}$} & \multirow{2}{*}{ p-valor* } \\
\hline & & Baixo & Moderado & Alto & & \\
\hline \multirow{2}{*}{$\begin{array}{l}\text { Doença } \\
\text { Pulmonar }\end{array}$} & Sim & $\begin{array}{c}8,33 \\
(n=1)\end{array}$ & $\begin{array}{l}8,33 \\
(n=1)\end{array}$ & $\begin{array}{c}83,34 \\
(n=10)\end{array}$ & $\begin{array}{l}100,00 \\
(n=12)\end{array}$ & \multirow{2}{*}{0,041} \\
\hline & Não & $\begin{array}{l}14,70 \\
(n=5)\end{array}$ & $41,18(n=14)$ & $\begin{array}{c}44,12 \\
(n=15)\end{array}$ & $\begin{array}{l}100,00 \\
(n=34)\end{array}$ & \\
\hline \multirow{2}{*}{ HAS } & Sim & $\begin{array}{l}6,90 \\
(n=2)\end{array}$ & $34,48(n=10)$ & $\begin{array}{l}58,62 \\
(n=17)\end{array}$ & $\begin{array}{l}100,00 \\
(n=29)\end{array}$ & \multirow{2}{*}{0,198} \\
\hline & Não & $\begin{array}{l}23,53 \\
(n=4)\end{array}$ & $29,41 \quad(n=5)$ & $\begin{array}{l}47,06 \\
(n=8)\end{array}$ & $\begin{array}{l}100,00 \\
(n=17)\end{array}$ & \\
\hline \multirow{2}{*}{ DM } & Sim & $\begin{array}{l}0,00 \\
(n=0)\end{array}$ & $\begin{array}{l}7,69 \\
(n=1)\end{array}$ & $\begin{array}{l}92,31 \\
(n=12)\end{array}$ & $\begin{array}{l}100,00 \\
(n=13)\end{array}$ & \multirow{2}{*}{0,002} \\
\hline & Não & $\begin{array}{l}18,18 \\
(n=6)\end{array}$ & $\begin{array}{c}42,43 \\
(n=14)\end{array}$ & $\begin{array}{l}39,39 \\
(n=13)\end{array}$ & $\begin{array}{l}100,00 \\
(n=33)\end{array}$ & \\
\hline
\end{tabular}

Nota: $(\mathrm{n}=46)$

p-valor $\leq$ 0,05 (aplicado teste do Qui-Quadrado).

Mesmo sem associação estatística, destacam-se as principais características dos participantes que apresentaram risco moderado e alto para quedas: sexo feminino, idade até 75 anos, tempo de internação de até dois dias e ter diagnóstico de HAS.

\section{DISCUSSÃO}

A média de idade encontrada neste estudo foi coerente com a esperança de vida ao nascer dos brasileiros que, atualmente, é de 76 anos $^{(13)}$. O risco de queda foi maior em idades mais elevadas, o que é corroborado com a literatura recente ${ }^{(7)}$.

A maioria dos participantes era do sexo feminino, perfil semelhante ao encontrado em outros estudos com idosos hospitalizados ${ }^{(9,11,14)}$. Tal característica pode ser explicada pelo fato de as mulheres idosas serem mais suscetíveis, por exemplo, ao desenvolvimento da síndrome da fragilidade na fase pós-menopausa, tornando-as mais vulneráveis a desfechos adversos ${ }^{(15)}$. Outros estudos identificaram que as quedas são mais frequentes no sexo feminino e estão associadas a um pior desempenho físico e histórico de doenças cardiovasculares ${ }^{(16-17)}$.

Predominou uma baixa escolaridade nesta amostra, fator que pode influenciar negativamente a qualidade de vida e, mais especificamente, na capacidade de compreender e adquirir informações relacionadas ao cuidado à saúde, já que outros autores identificaram que idosos com menor escolaridade tinham mais relatos de ocorrência de quedas ${ }^{(18)}$.

Nesta amostra, a internação por doença pulmonar teve associação significativa com o risco elevado de queda para os pacientes, dados que já haviam sido encontrados em pesquisas de outros autores ${ }^{(19)}$. Considerando que a Doença Pulmonar Obstrutiva Crônica (DPOC) tem como sintomas a dispneia e a fadiga aos esforços ${ }^{(20)}$, entende-se que essas alterações são fatores que podem contribuir para menor mobilidade, acarretando riscos de acidentes por quedas. Por outro lado, um estudo que investigou o risco de quedas em pacientes idosos com DPOC encontrou apenas 4,2\% da população com risco alto de quedas ${ }^{(21)}$.
Já o baixo risco de quedas teve associação significativa com os casos em que o motivo de internação era a realização de procedimento cirúrgico. No hospital estudado, as cirurgias realizadas são de pequeno e médio porte, de caráter eletivo - como, por exemplo, colecistectomia, herniorrafia e histerectomia -, por isso, a rotina é de que a alta hospitalar aconteça entre 24 e 48 horas, de modo que os pacientes costumam permanecer por pouco tempo na instituição.

Uma análise de coorte retrospectiva dos escores da Escala de Morse com mais de 8.000 pacientes hospitalizados encontrou associação entre maior tempo de permanência e maior frequência no grupo de risco para quedas ${ }^{(17)}$. Além disso, a internação hospitalar apresenta consequências sobre o estado físico e mental dos idosos. Como exemplo, destaca-se o delirium como o distúrbio neurocomportamental mais frequente em idosos hospitalizados, com a associação a elevados índices de queda ${ }^{(22)}$.

Estudo que avaliou risco de quedas entre pacientes adultos internados para procedimentos cirúrgicos não encontrou associação entre o porte da cirurgia e o risco de quedas, ao mesmo tempo em que identificou que, quanto mais avançada a idade, maior o risco elevado de quedas, bem como a presença de comorbidades como hipertensão, diabetes e câncer também estiveram associadas ${ }^{(23)}$. O fato de ter diabetes como comorbidade foi associado ao risco de quedas em mais outros dois estudos ${ }^{(23-24)}$.

Sobre isso, os idosos desta pesquisa que apresentavam diagnóstico médico de Diabetes Mellitus (DM) tiveram uma associação significativa com o risco elevado para quedas. Pesquisa realizada com idosos internados por fratura de quadril em decorrência de queda encontrou diabetes mellitus em $23,1 \%$ dos entrevistados, perdendo apenas para a hipertensão(25). Nessa perspectiva, necessita-se manter atenção maior sobre estes pacientes tendo em vista as potenciais complicações decorrentes da fisiopatologia dessa doença, tais como por nefropatia, retinopatia e lesões devido à neuropatia periférica.

A neuropatia periférica está associada a um pior desempenho na marcha e déficits de equilíbrio, limitações sensoriais e perda de força muscular, o que pode interferir nas tarefas 
cotidianas e, consequentemente, na qualidade de vida ${ }^{(24)}$. A ocorrência de quedas está associada também à presença de pisos escorregadios nas casas, perda auditiva, vertigem, hipertensão, uso de múltiplos medicamentos, depressão e incapacidade funcional ${ }^{(26)}$.

A hipertensão arterial apresentou-se como outra importante comorbidade ligada aos riscos moderado e elevado de queda. Pacientes hipertensos têm oitos vezes mais chances de queda do que os não hipertensos. Além disso, tanto a hipertensão quanto o uso de medicamentos anti-hipertensivos são fatores de risco para queda ${ }^{(23)}$.

O monitoramento dos episódios de quedas é de suma importância como método para subsidiar a formulação de medidas preventivas, admitindo orientar a gestão e as ações de cuidado focando na redução da ocorrência deste evento. Os estabelecimentos hospitalares devem estimular o desenvolvimento de recursos de trabalho que permitam e incentivem a identificação, a notificação e a análise deste evento adverso ${ }^{(3)}$.

Diante das características da população estudada e do expressivo número de pessoas com risco alto de quedas, entende-se que a equipe de enfermagem deve adotar práticas capazes de prevenir a ocorrência deste evento, tais como: comunicação efetiva entre profissionais de saúde e profissional/paciente; identificação do paciente com elevado risco por meio de sinalização à beira do leito ou pulseira com cor diferenciada; maior atenção ao movimentar os pacientes; alocar pacientes com alto risco para quedas em leitos mais próximos ao posto de enfermagem; apoio para realização de cuidados de higiene pessoal ${ }^{(27)}$.

Além dos cuidados diretos, também são importantes as ações de educação em saúde realizadas pelo profissional enfermeiro e outros profissionais de saúde para pacientes e acompanhantes, as quais devem incluir, por exemplo, orientações sobre a movimentação no leito e cuidados quando o idoso estiver fazendo uso de medicamentos com efeitos sedativos, pois são situações que favorecem a ocorrência de acidentes $^{(5,26)}$.

$\mathrm{O}$ investimento em equipamentos e utensílios como camas elétricas, barras de segurança nos banheiros e campainhas nos quartos, além da adoção de estratégias de sensibilização e orientação a pacientes, familiares e equipe de enfermagem, é capaz de diminuir a ocorrência do evento queda nas instituições. É importante também destacar o papel do enfermeiro nos programas de prevenção de quedas, pois este profissional pode avaliar o risco dos pacientes, planejar e implementar intervenções preventivas e, por fim, avaliar os resultados destas ${ }^{(28)}$, tendo como apoio a utilização de instrumentos como a Escala de Morse, usada no presente estudo.

O enfermeiro deve estimular a autonomia, a independência e a capacidade funcional dos idosos, reconhecendo seu papel na advocacia em saúde, objetivando a garantia da qualidade do cuidado, por ser o profissional presente continuamente no cuidado ao idoso hospitalizado, institucionalizado ou domiciliado. Ao desenvolver atividades de prevenção de quedas, o enfermeiro deve considerar também a avaliação do conhecimento do paciente e das intervenções educativas implementadas no ambiente hospitalar ${ }^{(29)}$.

Nesse sentido, uma recente revisão integrativa descreveu os indicadores para uma assistência de qualidade, que incluem: uso de calçado adequado, uso correto de dispositivos de assistência, iluminação do ambiente, adoção de procedimentos seguros de transferência, atenção para medicamentos prescritos ou não, condições crônicas e agudas que aumentem o risco de quedas, alterações na pressão arterial, entre outros. Adotando esses indicadores na avaliação de pacientes com o Diagnóstico de Enfermagem "Risco de quedas" em ambiente clínico real, o enfermeiro poderá examinar a efetividade das suas intervenções ${ }^{(30)}$.

O Diagnóstico de Enfermagem "Risco de quedas" possui mais de 40 intervenções descritas na Nursing Interventions Classification (NIC), mostrando a existência de inúmeros cuidados que o enfermeiro pode desempenhar com embasamento científico e que já possuem linguagem padronizada por meio de uma ferramenta de abrangência internacional ${ }^{(27)}$. A adoção dessa ferramenta pode então beneficiar a comunicação e minimizar a ocorrência de eventos adversos, reforçando a importância dos enfermeiros como promotores de ações de segurança do paciente.

$\mathrm{Na}$ condição de enfermeiras, é importante ressaltar que "Risco de quedas" é um diagnóstico de enfermagem aprovado há quase 20 anos, tendo sido atualizado na taxonomia vigente da North American Nursing Diagnosis Association (NANDA), em que foram definidas as populações em risco para tal evento, incluindo idade maior ou igual a 65 anos e história de quedas. Essa classificação aborda também as condições associadas a tal evento, que incluem: agente farmacêutico, alteração na função cognitiva, déficit proprioceptivo, doença aguda, doença vascular, equilíbrio prejudicado, hipotensão ortostática, entre outras.

Dentre as limitações desta pesquisa, destaca-se que, por tratar-se de um estudo transversal, os dados foram coletados em um único momento durante a internação dos participantes. Assim, possíveis alterações no estado de saúde ocorridas ao longo desse período não foram avaliadas, impedindo que outros fatores de risco potenciais fossem analisados.

Há necessidade de pesquisas posteriores que estudem a influência de tais condições sobre o evento quedas, como forma de fundamentar a elaboração de medidas que previnam sua ocorrência entre os idosos.

\section{CONCLUSÃO}

No presente estudo, mais da metade dos idosos apresentou risco alto para quedas. Tal situação variou de acordo com o motivo de internação e comorbidades referidas, sendo mais frequente naqueles internados por doença pulmonar e que apresentavam Diabetes Mellitus como comorbidade.

$\mathrm{O}$ risco alto para quedas em idosos hospitalizados encontrado no presente estudo mediante uso da Escala de Morse reforça a importância da aplicação desse instrumento na prática assistencial e a necessidade do desenvolvimento de estratégias de prevenção aos riscos e agravos do evento quedas no ambiente hospitalar. A adoção desse 
instrumento na prática assistencial tem como desafio o fato de ter sido introduzida no Brasil recentemente e, possivelmente, muitos profissionais de saúde ainda não a conheçam. Ações de educação permanente podem ser úteis para difundir esse conhecimento e estimular a adoção da Escala de Morse na rotina das instituições de saúde como uma medida importante para prevenção de eventos adversos em ambiente hospitalar, em conjunto com a implementação de outros protocolos institucionais de segurança do paciente.
Tais estratégias devem incluir todos os profissionais de saúde e gestores envolvidos no cuidado aos pacientes idosos hospitalizados, na realização de ações de educação em saúde, bem como na realização de adequações de leitos e enfermarias mais seguras para pacientes e acompanhantes, prevenindo a ocorrência do evento quedas. E, assim, evitando todas as repercussões negativas que esse agravo representa para a saúde da pessoa idosa e, ainda, os custos elevados com internações prolongadas em um serviço de saúde.

\section{RESUMO}

Objetivo: Identificar o risco de quedas em idosos em um hospital da região do Trairi, no Rio Grande do Norte; descrever a relação entre risco de quedas e as características sociodemográficas dos participantes. Método: Estudo descritivo, transversal, quantitativo, realizado em um hospital regional, respeitando os princípios éticos vigentes. Para a coleta, ocorrida em julho e agosto de 2018, foi adotada a Escala de Morse, adaptada com questões sociodemográficas. Realizada análise estatística descritiva e inferencial. Resultados: O estudo teve 46 participantes, dos quais a maioria era formada por mulheres, com baixa escolaridade e os motivos de internação mais frequentes foram tratamento cirúrgico e doença pulmonar. Mais da metade apresentou risco alto de sofrer quedas $(54,35 \%)$, seguido de moderado $(32,61 \%)$ e baixo $(13,04 \%)$. Houve associação entre risco alto de quedas, ter doença pulmonar como motivo de internação e diabetes como comorbidade. O risco alto de quedas foi menos frequente entre idosos internados para tratamento cirúrgico. Conclusão: $\mathrm{O}$ risco alto para quedas foi encontrado em mais da metade dos idosos, o que variou de acordo com o motivo de internação e comorbidades, sendo mais frequentes naqueles internados por doença pulmonar e que apresentavam Diabetes.

\section{DESCRITORES}

Idoso; Hospitalização; Acidentes por Quedas; Segurança do Paciente; Enfermagem Geriátrica.

\section{RESUMEN}

Objetivo: Identificar el riesgo de caídas en ancianos en un hospital de la región del Trairi, en Rio Grande do Norte; describir la relación entre riesgo de caídas y las características sociodemográficas de los participantes. Método: Estudio descriptivo, transversal, cuantitativo, realizado en un hospital regional, respetando los principios éticos vigentes. Para la recolección de datos, ocurrida en julio y agosto de 2018, fue adoptada la Escala de Morse, adaptada con cuestiones sociodemográficas. Realizado análisis estadística descriptiva y inferencial. Resultados: El estudio tuve 46 participantes, de los cuales la mayoría era formada por mujeres, con baja escolaridad y los motivos de internamiento más frecuentes fueran tratamiento quirúrgico y enfermedad pulmonar. Más de la mitad presento riesgo alto de sufrir caídas (54,35\%), seguido de moderado (32,61\%) y bajo (13,04\%). Hubo asociación entre riesgo alto de caídas, tener enfermedad pulmonar como motivo de internamiento y diabetes como comorbilidad. El riesgo alto de caídas fue menos frecuente entre ancianos internados para tratamiento quirúrgico. Conclusión: El riesgo alto para caídas fue encontrado en más de la mitad de los ancianos, lo que varió de acuerdo con el motivo de internamiento y comorbilidades, siendo más frecuentes en los internados por enfermedad pulmonar y que presentaban Diabetes.

\section{DESCRIPTORES}

Anciano; Hospitalización; Accidentes por Caídas; Seguridad del Paciente; Enfermería Geriátrica.

\section{REFERÊNCIAS}

1. Organização Mundial da Saúde; Direção-Geral de Saúde. Estrutura conceitual da classificação internacional sobre segurança do doente [Internet]. Lisboa: OMS; 2011 [citado 2018 mar. 09]. Disponível em: https://proqualis.net/sites/proqualis.net/files/Estrutura\%20 Conceitual\%20da\%20Classifica\%C3\%A7\%C3\%A3o\%20Int\%20Seguran\%C3\%A7a\%20do\%20Paciente.pdf

2. Rede Brasileira de Enfermagem e Segurança do Paciente. Estratégias para a segurança do paciente: manual para profissionais da saúde [Internet]. Porto Alegre: REBRAENSP; 2013 [citado 2018 mar. 08]. Disponível em: https://www.rebraensp.com.br/images/publicacoes/ manual_seguranca_paciente.pdf

3. Brasil. Ministério da Saúde; Agência Nacional de Vigilância Sanitária. Programa Nacional de Segurança do Paciente. Protocolo Prevenção de Quedas [Internet]. Brasília; 2013 [citado 2018 mar. 10]. Disponível em: https://www20.anvisa.gov.br/segurancadopaciente/index.php/ publicacoes/category/diversos

4. Sociedade Brasileira de Geriatria e Gerontologia. Projeto diretrizes: quedas em idosos: prevenção [Internet]. São Paulo: SBGG; 2008 [citado 2018 mar. 08]. Disponível em: https://www.projetodiretrizes. org.br/projeto_diretrizes/082.pdf

5. Vaccari E, Lenardt MH, Willig MH, Betiolli SE, Andrade LAS. Patient safety and falls in the hospital environment. Cogitare Enferm. 2016;21(n.spe):1-9. DOI: http://dx.doi.org/10.5380/ce.v21i5.45562

6. Gasparotto LPR, Falsarella GR, Coimbra AMV. Falls in elderly: basics concepts and updates of research in health. Rev Bras Geriatr Gerontol [Internet]. 2014 [cited 2018 Mar 10]; 17(1): 201-9. Available from: http://www.scielo.br/pdf/rbgg/v17n1/1809-9823-rbgg-17-01-00201.pdf

7. Cuevas-Trisan R. Balance problems and fall risks in the elderly. Phys Med Rehabil Clin N Am. 2017;28(4):727-37. DOI: 10.1016/j. pmr.2017.06.006

8. Morsch P, Myskiw M, Myskiw JC. Fall's problematization and risk factors identification through older adults narrative. Ciênc Saúde Coletiva. 2016;21(11):3565-74. DOI: 10.1590/1413-812320152111.06782016

9. Oliveira DU, Ercole FF, Melo LS, Matos SS, Campos CC, Fonseca EAM. Evaluation of falls in hospitalized elderly. Rev Enferm UFPE on line. 2017;11(11):4589-97. DOI: 10.5205/reuol.11138-99362-1-SM.1111sup201707 
10. Abreu DROM, Novaes ES, Oliveira RR, Mathias TAF, Marcon SS. Internação e mortalidade por quedas em idosos no Brasil: análise de tendência. Ciênc Saúde Coletiva. 2018; 23(4): 1131-1141. DOI: http://dx.doi.org/10.1590/1413-81232018234.09962016.

11. Sarges NA, Santos MIPO, Chaves EC. Evaluation of the safety of hospitalized older adults as for the risk of falls. Rev Bras Enferm [Internet]. 2017;70(4):860-7. DOI: http://dx.doi.org/10.1590/0034-7167-2017-0098

12. Urbanetto JS, Pasa TS, Bittencout HR, Franz F, Rosa VPP, Magnago TSBS. Analysis of risk prediction capability and validity of Morse Fall Scale Brazilian version. Rev Gaúcha Enferm. [Internet]. 2016 [cited 2019 Apr 19];37(4):e62200. Available from: http://www.scielo.br/ scielo.php?script=sci_arttext\&pid=S1983-14472016000400414\&lng=en.

13. Instituto Brasileiro de Geografia e Estatística. Tábua completa de mortalidade para o Brasil - 2017: breve análise da evolução da mortalidade no Brasil [Internet]. Rio de Janeiro: IBGE; 2018 [citado 2019 abr. 20]. Disponível em: https://biblioteca.ibge.gov.br/index.php/bibliotecacatalogo?view=detalhes\&id=2101628

14. Öztürk ZA, Özdemir S, Türkbeyler IH, Demir Z. Quality of Life and all risk in frail hospitalized elderly patients. Turk J Med Sc. 2017;47(5):1377-83. DOI: 10.3906/sag-1610-107

15. Fhon JRS, Rodrigues RAP, Neira WF, Huayta VMR, Robazzi MLCC. Fall and its association with the frailty syndrome in the elderly: systematic review with meta-analysis. Rev Esc Enferm USP. 2016;50(6):1005-13. DOI: http://dx.doi.org/10.1590/S0080-623420160000700018

16. Sharma PK, Bunker CH, Singh T, Ganguly E, Reddy PS, Newman AB, et al. Burden and correlates of falls among rural elders of South India: mobility and independent living in elders study. Current Gerontol Geriatr Res. 2017;2017:1290936. DOI: https://doi.org/10.1155/2017/1290936

17. Sardo PM, Simões CS, Alvarelhão JJ, Simões JF, Melo EM. Fall risk assessment: retrospective analysis of Morse Fall Scale scores in Portuguese hospitalized adult patients. Appl Nurs Res. 2016;31:34-40. DOI: https://doi.org/10.1016/j.apnr.2015.11.013

18. Cruz DT, Leite ICG. Falls and associated factors among elderly persons residing in the community. Rev Bras Geriatr Gerontol. 2018;21(5):551-61. DOI: http://dx.doi.org/10.1590/1981-22562018021.180034.

19. Oliveira CC, Lee AL, McGinley J, Thompson M, Louis BI, Anderson GP, et al. Falls by individual swith chronic obstructive pulmonary disease: a preliminary 12-month prospective cohort study. Respirology. 2015;20(7):1096-101. DOI: 10.1111/resp.12600

20. Lee J, Nguyen HQ, Jarrett ME, Mitchell PH, Pike KC, Fan VS. Effect of symptoms on physical performance in COPD. Heart Lung. 2018;47(2):149-56. DOI: 10.1016/j.hrtlng.2017.12.007

21. Fiel JNA, Lima JS, Dias JM, Neves LMT. Avaliação do risco de quedas e sarcopenia em idosos com doença pulmonar obstrutiva crônica atendidos em um hospital universitário de Belém, Estado do Pará, Brasil. Rev Pan Amaz Saúde. 2016;7(4):41-5. DOI: http://dx.doi.org/10.5123/s2176-62232016000400005

22. Faustino TN, Pedreira LC, Freitas YS, Silva RMO, Amaral JB. Prevention and monitoring of delirium in older adults: an educational intervention. Rev Bras Enferm [Internet]. 2016;69(4):725-32. DOI: http://dx.doi.org/10.1590/0034-7167.2016690416i

23. Mata LRF, Azevedo C, Policarpo AG, Moraes JT. Factors associated with the risk of fall in adults in the postoperative period: a cross-sectional study. Rev Latino Am Enfermagem. 2017;25:e2904. DOI: 10.1590/1518-8345.1775.2904

24. Bittencourt VLL, Graube SL, Stumm EMF, Battisti IDE, Loro MM, Winkelmann ER. Factors associated with the risk of falls in hospitalized adult patients. Rev Esc Enferm USP. 2017;51:e03237. DOI: 10.1590/S1980-220X2016037403237

25. Farias FID, Terra NL, Guerra MTE. Evaluation of the effectiveness of a care program for elderly patients with hip fractures: a network strategy. Rev Bras Geriatr Gerontol. 2017;20(5): 705-16. DOI: http://dx.doi.org/10.1590/1981-22562017020.170008

26. Jindal HA, Duggal M, Jamir L, Sharma D, Kankaria A, Rohilla L, et al. Mental health and environmental factors associated with falls in the elderly in North India: a naturalistic community study. Asian J Psychiatr. 2019;39:17-21. DOI: 10.1016/j.ajp.2018.11.013

27. Alves VC, Freitas Junior WC, Ramos JS, Chagas SRG, Azevedo C, Mata LRF. Actions of the fall prevention protocol: mapping with the classification of nursing interventions. Rev Latino Am Enfermagem. 2017;25:e2986. DOl: http://dx.doi.org/10.1590/1518-8345.2394.2986

28. Luzia MF, Cassola TP, Suzuki LM, Dias VLM, Pinho LB, Lucena AF. Incidence of falls and preventive actions in a University Hospital. Rev Esc Enferm USP. 2018;52:e03308. DOI: http://dx.doi.org/10.1590/S1980-220X2017024203308

29. Figueira AB, Barlem ELD, Tomaschewski-Barlem JG, Dalmolin GL, Amarijo CL, Ferreira AG. Actions for health advocacy and user empowerment by nurses of the Family Health Strategy. Rev Esc Enferm USP. 2018;52:e03337. DOI: http://dx.doi.org/10.1590/S1980-220X2017021603337

30. Luzia MF, Argenta C, Almeida MA, Lucena AF. Conceptual definitions of indicators for the nursing outcome "knowledge: fall prevention". Rev Bras Enferm [Internet]. 2018;71(2):431-9. DOI: http://dx.doi.org/10.1590/0034-7167-2016-0686 